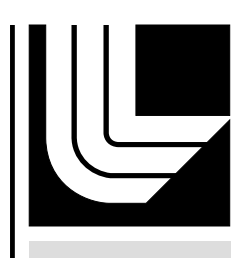

LAWRENCE LIVERMORE N A TION A L LABORATORY

\title{
High Energy Density Laboratory Astrophysics
}

B. A. Remington

November 30, 2004

12th International Conference on Plasma Physics Nice, France October 25, 2004 through October 31, 2004 
This document was prepared as an account of work sponsored by an agency of the United States Government. Neither the United States Government nor the University of California nor any of their employees, makes any warranty, express or implied, or assumes any legal liability or responsibility for the accuracy, completeness, or usefulness of any information, apparatus, product, or process disclosed, or represents that its use would not infringe privately owned rights. Reference herein to any specific commercial product, process, or service by trade name, trademark, manufacturer, or otherwise, does not necessarily constitute or imply its endorsement, recommendation, or favoring by the United States Government or the University of California. The views and opinions of authors expressed herein do not necessarily state or reflect those of the United States Government or the University of California, and shall not be used for advertising or product endorsement purposes. 


\title{
High energy density laboratory astrophysics
}

\author{
Bruce A. Remington ${ }^{a}$ \\ ${ }^{a}$ Lawrence Livermore National Laboratory
}

\begin{abstract}
High-energy-density (HED) physics refers broadly to the study of macroscopic collections of matter under extreme conditions of temperature and density. The experimental facilities most widely used for these studies are high-power lasers and magnetic-pinch generators. The HED physics pursued on these facilities is still in its infancy, yet new regimes of experimental science are emerging. Examples from astrophysics include work relevant to planetary interiors, supernovae, astrophysical jets, and accreting compact objects (such as neutron stars and black holes). In this paper, we will review a selection of recent results in this new field of HED laboratory astrophysics and provide a brief look ahead to the coming decade.
\end{abstract}

\section{INTRODUCTION}

Modern high power lasers and magnetic pinch facilities produce energy densities in millimeter-scale volumes large enough to access phenomena that otherwise appear only in energetic astrophysical systems. Examples of areas that can be studied include strong shock phenomena; high Mach number jets; strongly coupled plasmas; compressible hydrodynamic instabilities; radiation flow; photoevaporation front hydrodynamics; and fundamental properties such as opacities and equations of state. [Remington 99; 00]. Consequently, a new field of research is emerging - high energy 
density laboratory astrophysics. [HEDLA-2005 proceedings] We present a brief review of the emerging field of high energy density (HED) laboratory astrophysics, selecting experiments relevant to planetary interiors (Sec. II), core-collapse supernova explosion hydrodynamics (Sec. III), protostellar jet dynamics (Sec. IV), and accretion disk photoionized plasmas (Sec. V). A brief look ahead to the coming decade is given in Sec. VI.

\section{PLANETARY INTERIORS}

The interior structure of the giant planets of our solar system (Jupiter, Saturn, Uranus, and Neptune) is determined by the compressibility of their constituent matter under the very high pressures due to the inwardly directed force of gravity. [Guillot, 1999] In laboratory terms, this compressibility is determined by the equation of state (EOS) of the constituent matter along an isentrope [Saumon 2004]. The EOS and other properties of matter at the extreme pressures and densities found in the interiors of the giant planets, however, are quite uncertain. The pressures of interest along an isentrope range from 110 Mbar in Uranus and Neptune, [Hubbard, 1997] 1-40 Mbar in Saturn, to 1-70 Mbar in Jupiter, [Guillot 1999] as shown for Jupiter in Fig. 1a. Characteristic isentropes for the giant planets indicate that the plasma in their interiors is both strongly coupled $[\Gamma=$

$(\mathrm{Ze})^{2} / \mathrm{aT}>1$, where $\mathrm{Z}, \mathrm{e}, \mathrm{a}$, and $\mathrm{T}$ correspond to ionization state, electron charge, average atomic spacing, and temperature (in units of energy)] and degenerate $\left(\mathrm{T} / \varepsilon_{\mathrm{F}}<1\right.$, where $\varepsilon_{\mathrm{F}}$ is the Fermi energy). [Van Horn, 1991] Hence, the internal structure, $\rho(r), T(r)$, of the giant planets is determined by the EOS of dense, degenerate, strongly coupled plasmas and plasma mixtures at very high pressures, $\mathrm{P}=1$ to $40 \mathrm{Mbar}$, and moderate 
temperatures, $\mathrm{kT}<\sim 1 \mathrm{eV}$. The compressibility of hydrogen along a high pressure isentrope, and the predicted phase separation in a $\mathrm{He}-\mathrm{H}$ mixture when hydrogen transitions to a metallic state are of central interest, both for the giant planets of our solar system, and for models of the extrasolar planets. [Fortney, 2004]

A summary of recent experimental measurements of the shock-driven EOS of $\mathrm{D}_{2}$ at $~ 1$ Mbar pressures is given in Fig. 1b. [Knudson, 2003] Achieving $\sim$ Mbar pressures in these cryogenic hydrogen experiments is very difficult. The results from four different techniques are shown in Fig. 1b, corresponding to experiments done on a gas gun [Holmes, 1995], the Sandia Z facility [Knudson, 2003], a spherically convergent, HEdriven experiment [Be'lov, 2002; Boriskov, 2003], and the Nova laser [Collins, 1998]. The gas gun, Z, and $\mathrm{HE}$ results all more or less agree with each other. The gas gun and Z experiments both used the impedance matching technique, that measures the EOS of $\mathrm{D}_{2}$ relative to an assumed known EOS of Al. The Nova experiment was the only measurement in this set of four experiments that was absolute, that is, the compressibility of $\mathrm{D}_{2}$ was measured directly, as opposed to being inferred by comparison with the EOS of a reference material. This experiment showed enhanced compressibility, not evident in the other experiments. The reason for the difference between the Nova laser measurements, and all the rest of the measurements is still under debate. A new set of relative measurements done on the Omega laser favors an EOS of $\mathrm{D}_{2}$ that is less compressible than the Nova data, [Boehly, 2004] however, the situation is still far from settled. [Nellis, 2002; Mostovych, 2001] To conclude this ongoing debate, it seems likely that a new set of absolute measurements will be necessary.

There are a number of models of the EOS of high pressure hydrogen being 
compared with these data, as shown in Fig. 1b. The tabular SESAME EOS, and the $a b$ initio models generally are in agreement with the stiffer EOS data from Z. The linear mixing models and the Saumon-Chabrier-Van Horn (SCVH) model generally favor a softer EOS, that is, higher compressibility.

The impact on the predicted structure of the interior of Jupiter for the different EOS models is shown in Fig. 1c. [Saumon, 2004] This figure shows the mass of the core of Jupiter versus the mass of high-Z elements mixed throughout the planet, predicted using the five different models of high-pressure EOS of $\mathrm{D}_{2}$. Models that did not reproduce the global gravitational moments of Jupiter, as determined by satellite fly-by missions, were eliminated from these comparisons. The results in Fig. 1c show that the interior structure of Jupiter is surprisingly sensitive to the details of the high pressure EOS of hydrogen. Hence, there is considerable impact and scientific interest in concluding the experimental debate on the EOS of $\mathrm{D}_{2}$.

There are also new experimental results, measuring the EOS and conductivity of high pressure water, [Celliers, 2004; Koenig, 2004] relevant to the interior of Neptune. Additional new dynamic measurements address the EOS and high pressure melt transition in Fe, [Koenig, 2004; Koenig, 2000; Benuzzi-Mounaix, 2002; Nguyen, 2004] relevant to the inner-core, outer-core boundary region of the interior of earth.

\section{SUPERNOVAE}

Core-collapse supernovae result from the death of a massive star. Large stars have high enough temperatures in their cores to continue the nuclear fusion burning cycle up to Fe. Once the core reaches Fe, the nuclear fusion reactions no longer release net 
energy, and the thermonuclear fires are extinguished. At this point, there is no longer sufficient heat produced in the core to balance cooling by neutrino emission and photonuclear dissociation, and the core collapses under the force of gravity, triggering a catastrophic gravitational implosion that is over in a matter of seconds. This collapse is stopped only when the core density reaches that of degenerate nuclear matter $\left(\sim 2 \times 10^{14}\right.$ $\left.\mathrm{g} / \mathrm{cm}^{3}\right)$. The Fermi degeneracy pressure, $P_{\operatorname{deg}} \sim \rho^{2 / 3}$, increases sufficiently to stop the implosion, and a spectacular nuclear rebound occurs whose strength is determined by the EOS of bulk nuclear matter. By a mechanism still debated, this launches a powerful outward-propagating shock that eventually blows the star apart. This explosive birth is observed as a bright flash of UV light, followed by an extended period of enormous luminosity. If the core has a mass larger than $2-3 \mathrm{M}_{\mathrm{sun}}$, the core collapse continues to form a black hole, otherwise a neutron star is formed.

Once the core rebound shock gets launched, a number of nonlinear hydrodynamic effects are initiated. During the shock transit phase, the Richtmyer-Meshkov (RM) instability is triggered at each discontinuity in the density profile of the star, i.e., at the OHe and He-H "interfaces." After shock transit, hydrodynamic mixing continues due to the Rayleigh-Taylor (RT) instability, as the denser layers are decelerated by the lowerdensity outer layers. This deceleration is illustrated by the 1D simulation result (red dashed curve) in Fig.2a. [Kane, 1999] Large-scale, two-dimensional calculations of the development of the mixing at the O-He and $\mathrm{He}-\mathrm{H}$ interfaces [Kifonidis, 2000] show that spikes of dense, iron- and Ni-rich core material penetrate outward into the less dense envelope of hydrogen, but are slowed down abruptly by the reverse shock near the $\mathrm{H} / \mathrm{He}$ 
boundary (Fig. 2b). This interpenetration occurs through the growth and nonlinear evolution of the Rayleigh-Taylor (RT) instability.

Laser-based experiments can generate strong-shock induced nonlinear hydrodynamic mixing similar to those found in $\mathrm{SNe}$. In a set of experiments scaled to approximately reproduce the gross hydrodynamics of the He-H interface of SN1987A about an hour after explosion, a strong shock was passed through an interface separating dense "core" material $[\mathrm{CH}(4 \% \mathrm{Br})]$ from the lower density outer envelope (CRF foam). [Miles, 2004] A 2D ripple (1D wave vector) corresponding to an 8-mode sinusoidal pattern was imposed at the interface. The subsequent 2D growth due to the RM and RT instabilities was measured by $\mathrm{x}$-ray backlighting. Spikes of $\mathrm{CH}(\mathrm{Br})$ penetrating upward into less-dense $\mathrm{CH}_{2}$ as a consequence of the Rayleigh-Taylor instability were experimentally observed, and simulated in 2D with the radiation-hydrodynamics code CALE (Fig. 2c). The result of the simulations for an 8-mode perturbation at $13 \mathrm{~ns}$ is also shown in Fig. 2c, both without and with the addition of estimated experimental noise. The experimental result taken at the Omega laser is shown in Fig. 2c. The agreement between experiment and simulation at this level of (non-turbulent) nonlinear evolution is very good. Similar agreement was observed for single-mode and 2-mode 2D perturbations. [Miles, 2004] We conclude that in 2D, the nonlinear (non-turbulent) RT evolution can be very well reproduced with existing large-scale numerical simulations.

A theoretical look at the relation between the hydrodynamics occurring in the SN versus in the laboratory experiment shows that a rigorous mapping exists. In both settings, the Reynold's number (the ratio of the inertial to the viscous force) and the Peclet number (the ratio of the convective to the conductive heat transport) are large. 
Therefore, viscosity and thermal diffusivity are negligible, and the dynamics of the interface are well described by Euler's equations for a polytropic gas [Ryutov, 1999; 2000; 2001],

$$
\begin{aligned}
& \rho\left(\frac{\partial \mathbf{v}}{\partial t}+\mathbf{v} \cdot \nabla \mathbf{v}\right)=-\nabla p \\
& \frac{\partial \rho}{\partial t}+\nabla \cdot(\rho \mathbf{v})=0 \quad, \quad \text { and } \\
& \frac{\partial p}{\partial t}-\gamma \frac{p}{\rho} \frac{\partial \rho}{\partial t}+\mathbf{v} \cdot \nabla p-\gamma \frac{p}{\rho} \mathbf{v} \cdot \nabla \rho=0
\end{aligned}
$$

which represent conservation of momentum, mass, and entropy, respectively. It is straight-forward to show by substitution that Eq. 1 is invarient under the following scale transformation,

$$
\begin{aligned}
& \mathrm{h}_{\mathrm{SN}} \rightarrow \mathrm{ah} \text { lab, } \\
& \rho_{\mathrm{SN}} \rightarrow \mathrm{b} \rho_{\text {lab }}, \\
& \mathrm{p}_{\mathrm{SN}} \rightarrow \mathrm{cp}_{\text {lab }}, \\
& \tau_{\mathrm{SN}} \rightarrow \mathrm{a}(\mathrm{b} / \mathrm{c})^{1 / 2} \tau_{\text {lab }},
\end{aligned}
$$

where $h, \rho, p$, and $\tau$ correspond to characteristic spatial, density, pressure, and time scales, and subscripts $S N$ and $l a b$ refer to the supernova and laboratory laser experiment, respectively. When transformation (2) is inserted into Eq. (1), the constants a, b, and c cancel, and the dynamics described by Euler's equation are indistinguishable in the SN and the laser experiment. Both settings are probing the same physics. Any insights 
gained through the laser experiment apply directly to the $\mathrm{SN}$ through the mapping described by Eq. 2. For example, the hydrodynamics illustrated in Figs. 2 b and $2 c$ are similar, and can be related through the $\mathrm{SN}$-to-laboratory mapping of $\mathrm{h}, \rho, \mathrm{p}, \tau$, and $\mathrm{g}=$ $\nabla \mathrm{p} / \rho$, given by Eq. 2. [Ryutov, 1999]

There are additionally a number of experiments measuring strong shock and radiative shock dynamics relevant to supernova remnants (SNRs). [Keiter, 2002; Reighard, 2004; Hansen, 2004; Fleury, 2002; Edwards, 2001; Bouquet, 2004] Analytic theory for astrophysical and laboratory radiative shocks and radiative precursor shocks has also been developed. [Bouquet, 2000; Michaut, 2004a; Michaut, 2004b; Keilty, 2000]

\section{PROTOSTELLAR JETS}

Astrophysical jets present some of the most visually intriguing images that we encounter in the galaxy and universe. [Reipurth, 2001] These jets cover an enormous range of spatial scale, from $10^{17} \mathrm{~cm}$ for jets from young stellar objects (see Fig. 3a) to $10^{24} \mathrm{~cm}$ for jets associated with quasars or active galactic nuclei (AGN) harboring massive black holes (see Fig. 4a). The class of stellar jets known as Herbig-Haro (HH) objects are thought to be collimated bipolar outflows emerging from accretion disks during the star formation process. An example of a Herbig-Haro jet, $\mathrm{HH} 111$, is shown in Fig. 3a. Typical velocities of the HH-jets are a few hundred $\mathrm{km} / \mathrm{s}$ at densities of $\mathrm{n}_{\text {jet }}=10$

$-100 \mathrm{~cm}^{-3}$. In terms of density contrast, this corresponds to $\eta=\mathrm{n}_{\mathrm{jet}} / \mathrm{n}_{\text {ambient }}=0.01-10$, depending on the density of the ambient medium, where $\mathrm{n}_{\text {jet }}$ and $\mathrm{n}_{\text {ambient }}$ are the jet and ambient number density, respectively. [Blondin, 1990] Such high Mach-number, high density jets can often be radiatively cooled. 
Models of jet formation generally fall into two broad categories. One category assumes that jets are formed as a consequence of matter falling into a compact central object from a magnetized rotating accretion disk. In the case of $\mathrm{HH}$ jets, the compact central object is the protostar or young stellar object (YSO). A second class of models assumes that the accretion process onto a central object results in a toroidal accretion disk, with density maximum along the equator and minimum at the poles, which define the symmetry (and rotation) axis. Then spherically symmetric, outflowing winds from the central object interact with this toroidal density profile, which leads to a funneling of the wind into bipolar jets. Observational data are insufficient at this time to conclusively discriminate between these two classes of models, and indeed both may be operating simultaneously. An additional complexity in modeling high Mach-\# jets such as the $\mathrm{HH}$ jets is that they can be radiatvbely cooled. This lowers the pressure inside the jet, and leads them to compress further, due to the pressure of the shocked ambient medium, leading to denser, more collimated jets, such as shown by the simulations in Fig. $3 b$. [Blondin, 1990] As the radiative cooling increases, as quantified by the cooling parameter, $\chi=\mathrm{L}_{\text {cool }} / \mathrm{R}_{\text {jet }}$, the jet density and collimation increases. A central question concerns how these jets remain so well collimated over propagation distances exceeding $\sim 10$ jet diameters.

An emerging new experimental capability may prove useful in testing aspects of these models in addressing the collimation question. With the development of sophisticated high power lasers and magnetic pinch facilities around the world, the ability to conduct well controlled and well diagnosed laboratory experiments on energetic jets is now possible. Laboratory experiments allow the effects of high Mach number, radiative 
cooling, and possibly magnetic fields on the jet dynamics to be probed individually or in combinations. The potential benefit of this research to astrophysics is only starting to be recognized and pursued.

One example, done on the Omega laser at the University of Rochester, is a purely hydrodynamic, high Mach number jet, shown at two different times in Fig. 3c. [Foster, 2002] In the experiment, a set of 12 laser beams at a total energy of $\sim 6 \mathrm{~kJ}$ enters a cylindrical gold cavity (hohlraum) on one end through the laser entrance hole (LEH), and converts to $\mathrm{x}$-rays at or near the inner gold wall surface, generating a $\mathrm{T}_{\mathrm{r}} \approx 200 \mathrm{eV}$ radiation burst which lasts of order $\sim 1 \mathrm{~ns}$. A solid Al cylindrical plug of dimensions 200 $\mu \mathrm{m}$ diameter by $150 \mu \mathrm{m}$ length is mounted on a hole on the wall of the hohlraum opposite the LEH, with $100 \mu \mathrm{m}$ of the Al protruding into the hohlraum. This Al cylinder is butted up against a $\sim 1 \mathrm{~mm}$ long tube filled with a solid-density plastic $(\mathrm{CH})$ reservoir. The radiation bath in the hohlraum ablatively launches converging shocks into the $\mathrm{Al}$ cylindrical plug. The magnitudes of the observed early-time jet and shock velocities suggest that an initial, very high pressure region in the center of the Al cylinder, $\mathrm{P}>100$ Mbar (ie, $10^{14}$ dyne $/ \mathrm{cm}^{2}$ ), is created. The Al-CH interface at the entrance to the $\mathrm{CH}$ reservoir is at ambient pressure, hence, the high internal pressure in the Al launches a high Mach number jet of $\mathrm{Al}$ axially down the $\mathrm{CH}$ tube. This jet is imaged radiographically, by generating a timed burst of hard x-rays behind the target, and imaging the jet as a shadow (to the "backlighter" x-rays). This external source of backlighter x-rays is generated by focussing several of the Omega beams onto an auxillary planar disk of material such as Ti, generating a bright source of $\mathrm{He}-\alpha \mathrm{X}$-rays at $4.7 \mathrm{keV}$. The jet shown in Fig. 3c has moved about 3 jet diameters, and a very 
prominent Kelvin-Helmholtz $(\mathrm{KH})$ rollup is observed at the jet tip. The bow shock in the plastic is also very evident in these images. Simulations of the experiment are shown in the top of Fig. 3c, and the experimental data are at the bottom of the figure. The internal Mach numbers in these jets are $\mathrm{M}_{\mathrm{int}}=\mathrm{v}_{\mathrm{jet}} / \mathrm{c}_{\mathrm{s}}(\mathrm{jet}) \approx 3$, whereas the external Mach numbers are $\mathrm{M}_{\text {ext }}=\mathrm{v}_{\text {jel }} / \mathrm{c}_{\mathrm{s}}($ ambient $) \approx 5$. The density ratio at the time off the images is $\eta=$ $\rho_{\mathrm{jer}} / \rho_{\mathrm{ambient}} \sim 0.3$, due to the decompression of the $\mathrm{Al}$, and the shock compression of the plastic. Once launched, these jets were purely hydrodynamic, with effects due to radiation being insignificant other than generating the initial ablation pressure source. For hydrodynamic jets such as these, a rigorous scale transformation can relate the laboratory experiments to the astrophysical setting. [See scaling discussion in Sec. III above.]

A very attractive alternate way to produce scaled, high Mach-\# jets is to use a magnetic pinch facility, such as the Magpie Z-pinch at Imperial College, UK. In this technique, a fast-rising current (reaching $1 \mathrm{MA}$ in $240 \mathrm{~ns}$ ) is applied to a conical array of fine metallic wires. [Lebedev, 2002] The resistive heating of the wires by the current rapidly converts the outer surfaces of the wires to plasma, and through the J X B force, a fraction of this plasma implodes onto the axis. Due to the initial conical shape of the wires, the imploded "precursor" plasma has a net axial velocity component, and turns into a jet. If the wires are high $\mathrm{Z}$, such as tungsten, then the jet is radiatively cooled. As the precursor plasma stagnates on axis, the jet radiatively cools and remains better collimated as it emerges from the pinch regions. This high Mach-\# jet can then be impacted into an ambient medium, such as a $\mathrm{CH}$ plasma target. During this impact the jet tip region ("working surface") reheats, and starts to radiate in the soft x-ray again. An example of such an impact from jet experiments done on the Magpie pinch facility is shown in Fig. 3d. [Lebedev, 2002] 


\section{ACCRETION DISK PHOTOIONIZED PLASMAS}

One of the most intriguing objects in the universe is an accreting compact object, such as a neutron star or black hole. At the extreme is an accretion disk around a massive black hole $\left(10^{8}-10^{9} \mathrm{M}_{\text {sun }}\right)$ at the center of a galaxy such as the active galactic nucleus (AGN) object NGC 4261. [Ferrarese, 1996] The accreting, massive black hole also generates bipolar intergalactic jets, which taken together form a quasar. [Pinar, 2001]. Observational evidence has been presented for a massive black hole in the active galaxy NGC 4261, a giant elliptical galaxy at a distance of $30 \mathrm{Mpc}$, from HST images.

[Ferrarese, 1996] From an analysis of optical images obtained using HST/WFPC2, similar to that shown in Fig. 4a, it is deduced that the ionized gas near the center is in circular motion, consistent with a central mass of $\sim 4.9 \times 10^{8} \mathrm{M}_{8}$ inside of a spatial extent of $15 \mathrm{pc}$. The corresponding mass-to-light ratio is $(\mathrm{M} / \mathrm{L}) \sim 2 \times 10^{3} \mathrm{M}_{8} / \mathrm{L}_{8}$, supporting the interpretation of a central massive black hole. There appears to be an intimate connection between the dust disk and the central engine, which drives the two bipolar radiojets of $10^{5}$ lgt yr extent that eminate normally from the disk. [Pinar, 2001] The inner dust disk is thought to be supplying fuel to the central engine, and an outer dust disk is thought to be feeding fuel to the inner accretion disk that surrounds the massive black hole.

Another much closer example is Cyg X-3, an accreting x-ray binary system. An example spectrum from this accreting binary $x$-ray source Cyg X-3 is shown in Fig. 4 b. [Liedahl, 1996] These spectra result from the final plunge of matter from the accretion disk into the compact object, and have been shown to result from a photoionized plasma. In this case, radiative excitation, absorption, and emission processes dominate, and 
collisional processes are negligible. The emission-line spectrum of the X-ray binary Cygnus X-3 is consistent with recombination-dominated line formation. From this it is inferred that the source of energy "pumping" the lines is the hard X-ray continuum. The simplest interpretation of Cyg X-3 assumes that the x-ray emission is from plasma in photoionization equilibrium.

To check or calibrate the models used to interpret these spectra, experimental data of photoionzied plasmas in relevant regimes are required. It was recognized recently that similar conditions of photoionized plasmas could be created in the laboratory using the intense burst of x-rays coming from the z-pinch at the SNLA Z-facility. [Heeter, 2001] An experiment was developed to measure the photoionized plasma x-ray spectra under approximately scaled conditions. The figure of merit, the ionization parameter $\xi=\mathrm{L} / \mathrm{n}_{\mathrm{e}} \mathrm{r}^{2}$, where $\mathrm{L}, \mathrm{n}_{\mathrm{e}}$, and $\mathrm{r}$ are the ionizing (x-ray) luminosity, electron density, and distance from the central source of ionizing radiation, resp., needs to be large, $\xi \geq 100$, to be relevant to astrophysical photoionized plasmas. This implies radiation dominance in the excitation and de-excitation processes.

The experiments were performed at the Sandia National Laboratory $Z$ facility. The radiation from the pinch was generated by coupling a $20 \mathrm{MA}, 100 \mathrm{~ns}$ rise time current pulse into a $2 \mathrm{~cm}$ diameter, $1 \mathrm{~cm}$ length, cylindrical wire array, creating a $8 \mathrm{~ns}$ FWHM, $120 \mathrm{TW}, \mathrm{T}_{\mathrm{r}}=165 \mathrm{eV}$ near-blackbody radiation source. The sample charge state distribution, the absolute radiative flux, and the sample densities were measured independently. A typical spectrum from an Fe sample, located a distance of $1.5-1.6 \mathrm{~cm}$ from the pinch, is shown in Fig. 4c. [Foord, 2004] In this experiment, $\xi$ reaches a value 
near $25 \mathrm{erg} \mathrm{cm} / \mathrm{s}$ at the peak of the radiation pulse, close to the desired values of $10^{2}-10^{3}$ to resemble those of an accreting black hole.

A number of photoionized plasma models have now been compared with this laboratory experiment. Comparison of the predicted versus experimentally observed ionization distribution for an iron plasma is shown in Fig. 4d. [Rose, 2004] A radiation temperature of $T_{r}=165 \mathrm{eV}$, electron temperature of $T_{e}=150 \mathrm{eV}$ and electron number density of $\mathrm{n}_{\mathrm{e}}=2 \times 10^{19} \mathrm{~cm}^{-3}$ [Foord et al 2004] were assumed for the calculations with the average-atom model NIMP. Calculations are shown with and without including the radiation field. The observed ionization state would be underpredicted without the inclusion of the radiation field, which is a central feature of astrophysical photoionized plasmas. Also included in the comparison are predictions from the more detailed model GALAXY presented for the same conditions.

Good agreement with experiment is found only for the calculations that include the radiation field, but collisional effects are not completely negligible. The averageatom model is observed to be quite effective at calculating these photoionized plasma $\mathrm{X}$ ray spectra, which is an important conclusion, because of its wide use in modeling laboratory plasmas.

\section{CONCLUSION}

Looking ahead, there will be unique regimes of HED laboratory astrophysics that will become accessible on the NIF laser, under construction in the U.S. [Hogan, 2001], and the LMJ laser under construction in France. [Andre, 1999] The key scientific uncertainties in hydrogen relative to planetary interiors are at pressures greater than 1 
Mbar, and along a quasi-isentrope. With the NIF and LMJ lasers, quasi-isentropic compression of hydrogen and $\mathrm{H}-\mathrm{He}$ mixtures to pressures considerably greater than 1 Mbar should be possible, allowing tests of high pressure EOS models under conditions most relevant to the interiors of the giant planets.

In the studies of supernova explosion hydrodynamics, the key issues to examine are, in scaled divergent geometry, an experiment that evolves fully into the turbulent regime, in a diagnosable configuration. With the large energies and pointing flexibility of the NIF and LMJ lasers, such a scaled supernova hydrodynamics experiment should be both possible and diagnosable. This would help answer whether the "standard model" of core collapse supernovae is able to reproduce the astronomically observed rapid core inversions.

One of the key issues in protostellar jet dynamics is how high Mach number, radiatively cooled jets stay collimated, and under what conditions they do or do not become turbulent, in their interactions with the interstellar medium (ISM). Scaled jet experiments on the NIF and LMJ lasers should be able to evolve deeply into regimes where turbulence should be expected, and thereby help answer questions relevant to the collimation of protostellar jets.

To study scaled systems relevant to compact object (neutron stars, black holes) accretion disks requires a low density, radiation dominated, photoionized plasma. An exceedingly high flux of thermal x-rays is needed so that, in the surrounding plasma, atomic excitations, ionization, and recombination processes are dominated by the radiation field, with the effects from electron-ion collisions being negligible. On NIF and LMJ, creating these intense $\mathrm{x}$-ray luminosities should be possible, allowing models of 
photoionized plasmas, relevant to accreting black holes, to be checked and calibrated, in a properly scaled experimental testbed.

In conclusion, exceptional progress has been made over the past decade on developing the new field of HED laboratory astrophysics, as is evident by the breadth and depth of results being reported in literature. [HEDLA-2004, 2005] With the construction of the NIF and LMJ lasers, the next decade should witness several breakthroughs in understanding of energetic astrophysical processes, aided by scaled HED laboratory experiments.

\section{ACKNOWLEDGEMENTS}

*The work was carried out under the auspices of the U.S. Department of Energy by the Lawrence Livermore National Laboratory under contract number W-7405-ENG-48.

\section{REFERENCES}

[Andre, 1999] M.L. Andre, Fusion Engineering and Design 44: 43-49 FEB 1999 [Belov, 2002] S.I. Belov et al., Pis'ma Zh. Eksp. Teor. Fiz. 76, 508 (2002) [English version: JETP Lett. 76, 433 [2002)].

[Benuzzi-Mounaix, 2002] A. Benuzzi-Mounaix et al.., Phys. Plasmas 9, 2466 (2002). [Blondin, 1990] J. Blondin et al., Ap. J. 360, 370 (1990). [Boehly, 2004] T. Boehly et al., Phys. Plasmas 11, L49 (2004).

[Bouquet, 2000] S. Bouquet et al., Ap. J. Suppl. 127, 245 (2000).

[Bouquet, 2004] S. Bouquet et al., Phys. Rev. Lett. 92, 225001 (2004).

[Boriskov, 2003] G.V. Boriskov et al., Dokl. Akad. Nauk 392, 755 (2003) [English version: Dokl. Phys. 48, 553 (2003).

[Celliers, 2004] P.M. Celliers et al., Phys. Plasmas 11, L41 (2004).

[Collins, 1998] G.W. Collins et al., Science 281, 1178 (1998).

[Edwards, 2001] J. Edwards et al., Phys. Rev. Lett. 87, 085004 (2001).

[Ferrarese, 1996] Ferrarese et al., Ap. J. 470, 444 (1996).

[Fleury, 2002] X. Fleury et al., Laser and Particle Beams 20, 263 (2002).

[Foord, 2004] M.E. Foord et al., Phys. Rev. Lett. 93,, 055002 (2004).

[Fortney, 2004] J.J. Fortney and W.B. Hubbard, Åp. J. 608, 1039 (2004).

[Foster, 2002] J. Foster et al., Phys. Plasmas 9, 2251 (2002).

[Guillot, 1999] T. Guillot, Science 286, 72 (1999). 
[Hansen, 2004] J.F. Hansen et al., submitted, Phys. Rev. Lett. (2004).

[HEDLA 05] Proceedings of the 5th International Conference on High Energy Density

Laboratory Astrophysics, in press, Astrophysics and Space Science 298:1-2 (July 2005).

[Heeter, 2001] R.F. Heeter et al., Rev. Sci. Instrum. 72, 1224 (2001).

[Hogan, 2001] W.J. Hogan et al., Nuclear Fusion 41, 567 (2001).

[Holmes, 1995] N. Holmes et al.,PRB 52, 15835 (1995).

[Hubbard, 1997] W.B. Hubbard, Science 275, Issue 5304, 1279 (1997).

[Kane, 1999] J. Kane et al., PoP 6, 2065 (1999).

[Keilty, 2000] K.A. Keilty et al., Ap. J. 538, 645 (2000).

[Keiter, 2002] P.A. Keiter et al., Phys. Rev. Lett. 89, 165003 (2002).

[Kifonidis, 2000] Kifonidis et al., Ap. J. 531, L123 (2000).

[Knudson, 2003] M.D. Knudson et al., PRL 90, 035505 (2003).

[Koenig, 2004] M. Koenig et al., Nuclear Fusion, in press (2004).

[Koenig, 2000] M. Koenig et al., Recherche 330, 46 (2000).

[Lebedev, 2002] S. Lebedev et al., Ap.J. 564, 113 (2002).

[Liedahl, 1996] D.A. Liedahl and F. Paerels, Ap. J. 468, L33 (1996).

[Michaut, 2004a] C. Michaut et al., IFSA-2003 proceedings (2004).

[Michaut, 2004b] C. Michaut et al., Eur. Phys. J. D 28, 381 (2004).

[Miles, 2004] A. Miles et al., PoP 11, 3631 (2004).

[Mostovych, 2001] A.N. Mostovych et al., Phys. Plasmas 8, 2281 (2001).

[Nellis, 2002] W.J. Nellis et al., Phys. Rev. Lett. 89, 165502 (2002).

[Nguyen, 2004] J.H. Nguyen and N.C. Holmes, Nature 427, 339 (2004).

[Piner, 2001] Piner et al., A.J. 122, 2954 (2001).

[Reighard, 2004] A.B. Reighard et al., submitted, Phys. Rev. Lett. (2004).

[Reipurth, 2001] B. Reipurth and Bally, Annual Reviews of Astronomy and Astrophysics 39, 403 (2001).

[Remington, 1999] B.A. Remington et al., Science 284, 1488 (1999).

[Remington, 2000] B.A. Remington et al., Phys. Plasmas 7, 1641 (2000).

[Rose, 2004] S.J. Rose et al., J. Phys. B: At. Mol. Opt. Phys. 37, L337 (2004).

[Ryutov, 1999] D.D. Ryutov et al., Ap. J. 518, 821 (1999).

[Ryutov, 2000] D.D. Ryutov et al., Ap. J. Supple. 127, 465 (2000).

[Ryutov, 2001] D.D. Ryutov et al., Phys. Plasmas 8, 1804 (2001).

[Saumon, 2004] D. Saumon and T. Guillot, Ap.J. 609,1170 (2004).

[Van Horn, 1991] H.M. Van Horn, Science 252, 384 (1991).

\section{FIGURE CAPTIONS}

Fig. 1. (a) Schematic of the interior of Jupiter, reproduced from [Guillot, 1999]. (b) Summary plot of recent high pressure shock Hugoniot measurements of $\mathrm{D}_{2}$, reproduced from [Knudson, 2003]. (c) Plot showing the sensitivity of calculations of the interior of Jupiter to the models of the high pressure EOS of hydrogen, reproduced from [Saumon, 2004]. The different curves correspond to the following models. LM-A: Modified Ross linear mixing model. LM-H4: Linear mixing, with $\mathrm{D}_{4}$ chains, $\mathrm{D}_{2}$ molecules, and a $\mathrm{D}^{+}+\mathrm{e}^{-}$ fluid metal. LM-SOCP: Ross linear mixing model, only with the One Component Plasma (OCP) replaced with a screened OCP for the metallic (atomic) fluid free energy. 
SCVH-1: the Saumon-Chabrier, Van Horn EOS. SESAME-p: original SESAME, patched to better reproduce data in the molecular regime for $\mathrm{P}<0.2 \mathrm{Mbar}$.

Fig. 2. (a) 1D simulation showing the "interface" velocity for a supernova explosion, corresponding to the $\mathrm{He}-\mathrm{H}$ interface, and the $\mathrm{Cu}-\mathrm{CH}$ interface of a scaled laser target experiment. Reproduced from [Kane, 1999]. (b) 2D simulation of SN 1987A, showing the RT instability induced spikes of core material impacting the reverse shock at the HeH interface. Reproduced from [Kifonidis, 2000]. (c) Simulations, without and with, the addition of an estimate of the experimental noise level, compared with the actual experiment, for the nonlinear RT evolution of a preimposed 8-mode perturbation. Reproduced from [Miles, 2004].

Fig. 3. (a) Optical image of Herbig-Haro $(\mathrm{HH})$ jet 111, reproduced from [Reipurth, 2001]. (b) Simulations of high Mach number jets relevant to $\mathrm{HH}$ jets, showing the sensitivity to the amount of radiative cooling, reproduced from [Blondin, 1990]. (c) Simulations, and experimental images of a high Mach number, purely hydrodynamic (non-radiatively cooled) Al jet experiment done on the Omega laser, located at the University of Rochester. Reproduced from [Foster, 2002]. (d) Experimental image of a high Mach number, radiatively cooled W jet, done on the Magpie z-pinch facility at Imperical College. Reproduced from [Lebedev, 2002].

Fig. 4. (a) Images of the intergalactic jet, and the accretion disk around the active galactic nucleus, NGC 4261, reproduced from [Piner, 2001; Ferrarese, 1996]. (b) X-ray spectrum of the photoionized plasma in the immediate vicinity of Cyg X-3 x-ray binary, reproduced from [Liedahl, 1996]. (c) X-ray spectrum in a scaled experiment done on the Z pinch facility at SNLA, reproduced from [Foord, 2004]. (d) Photoionized plasma models of the ionization distribution observed in the $\mathrm{Z}$ photoionized plasma experiment, reproduced from [Rose, 2004]. 

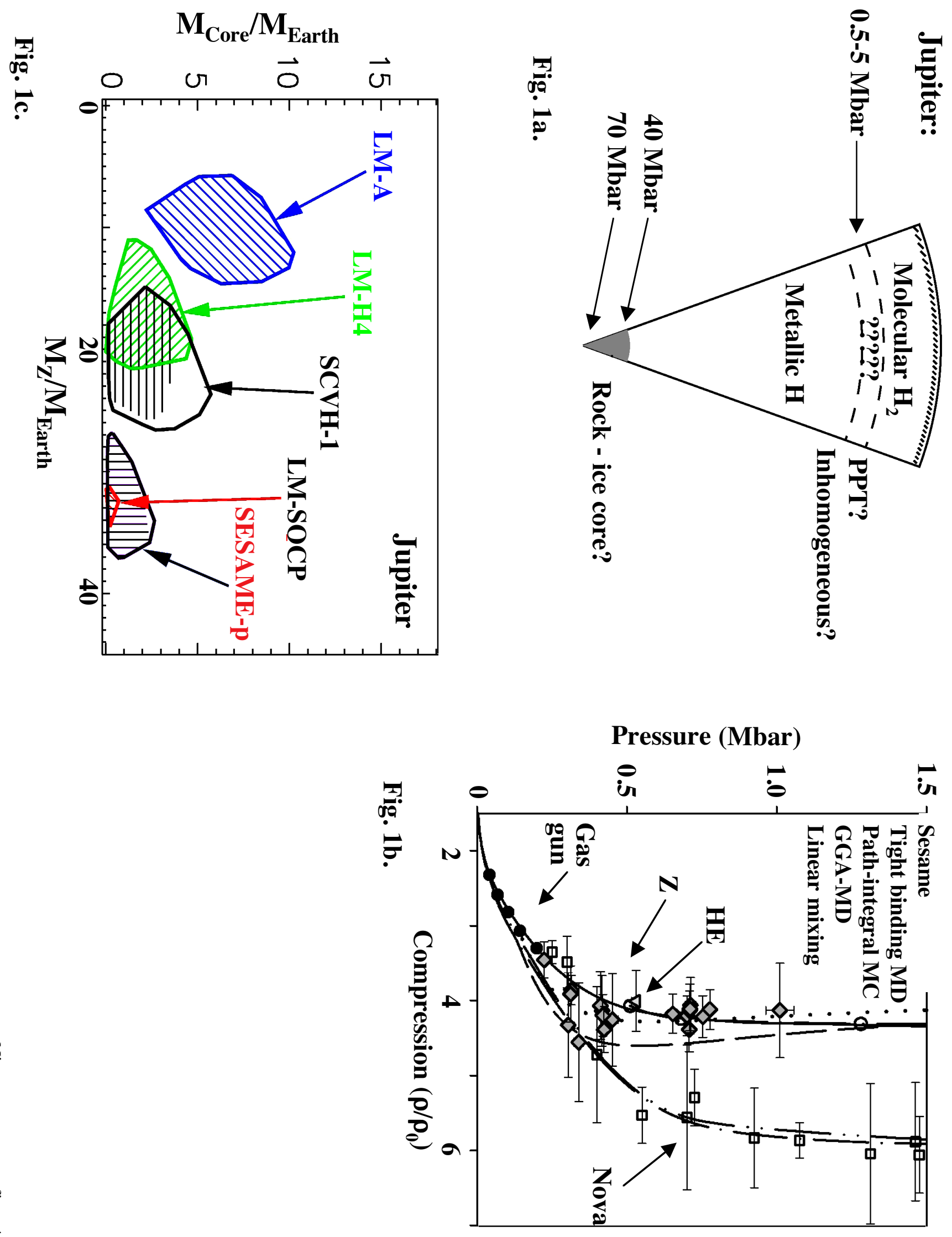

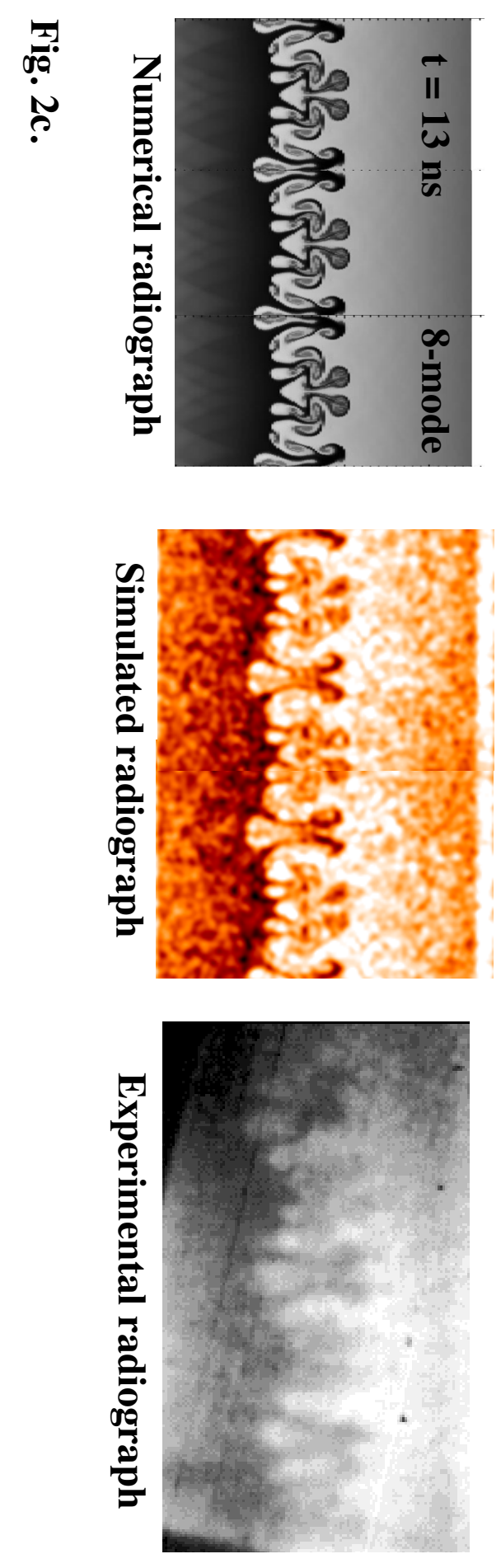
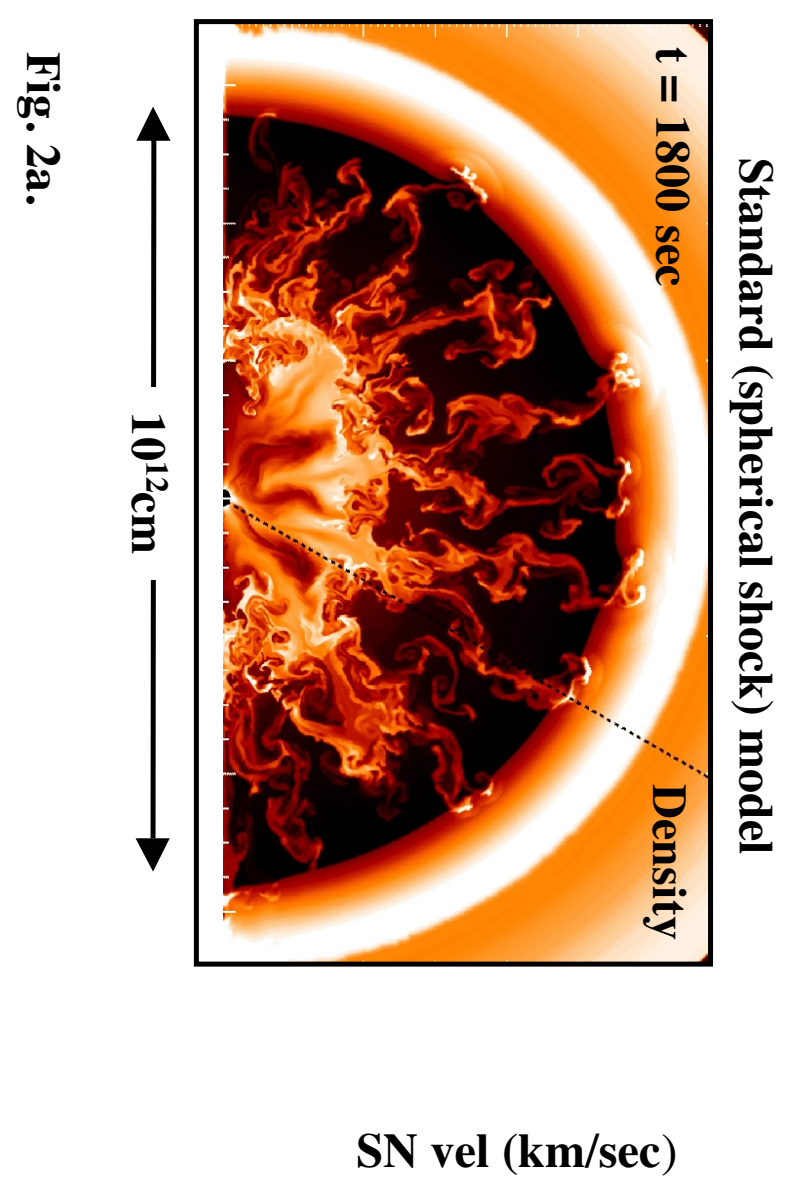

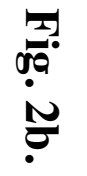

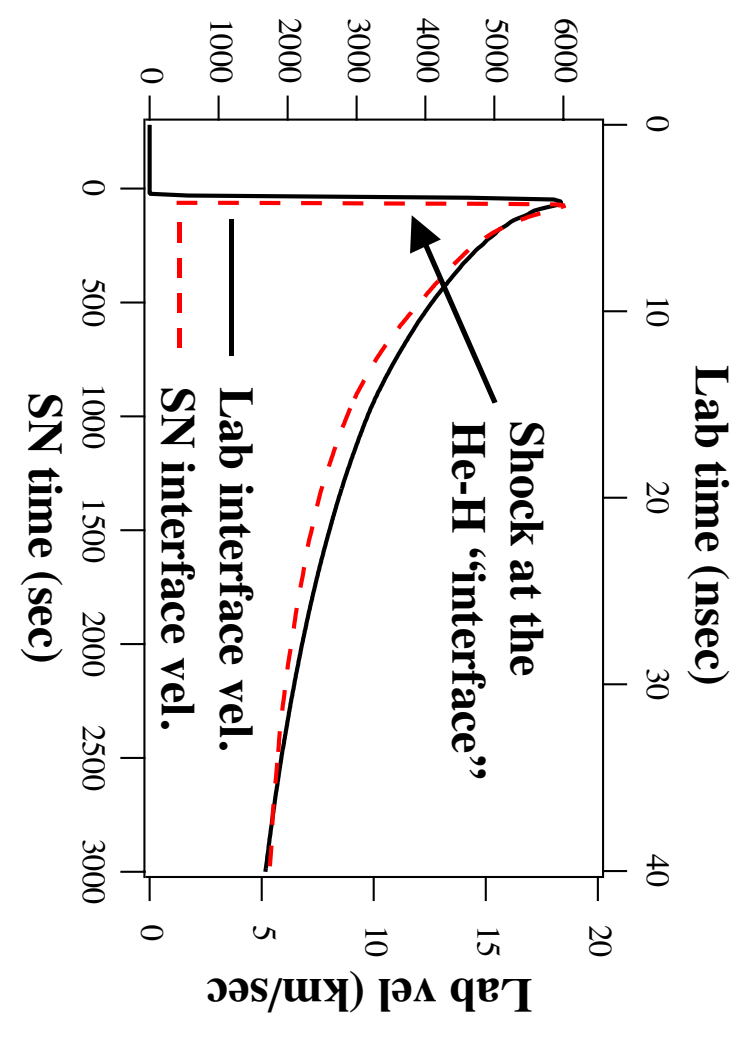



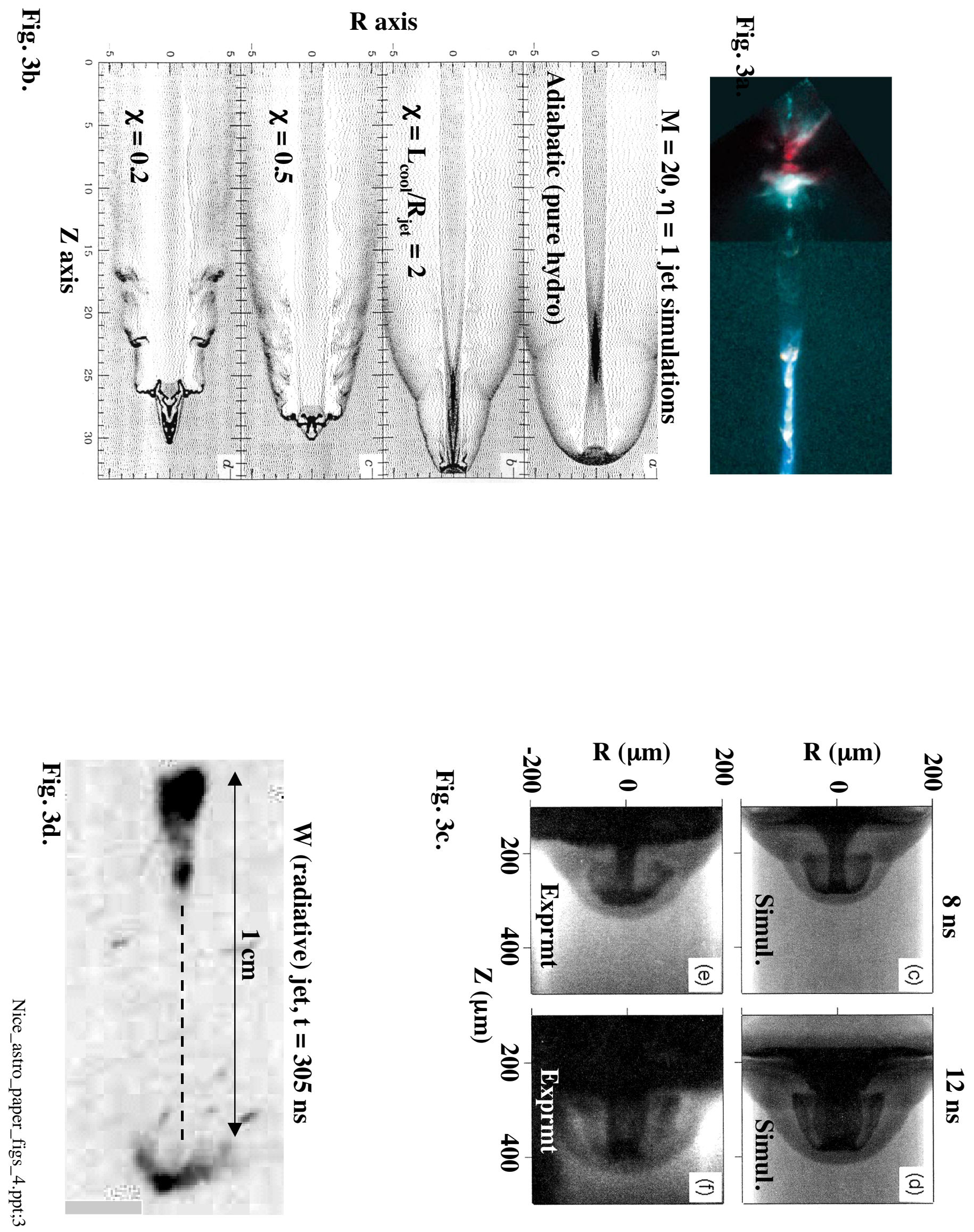

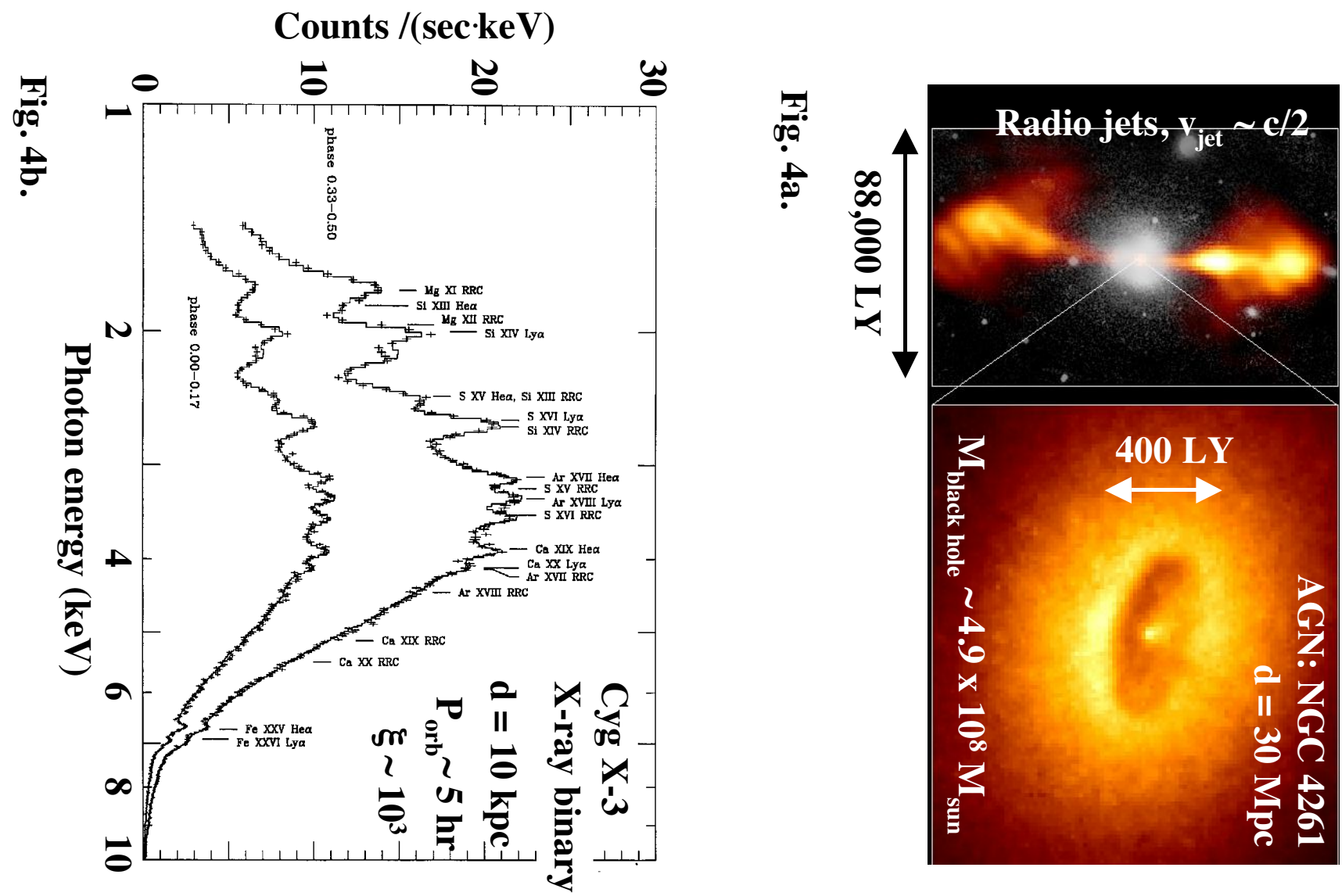

Charge state fraction
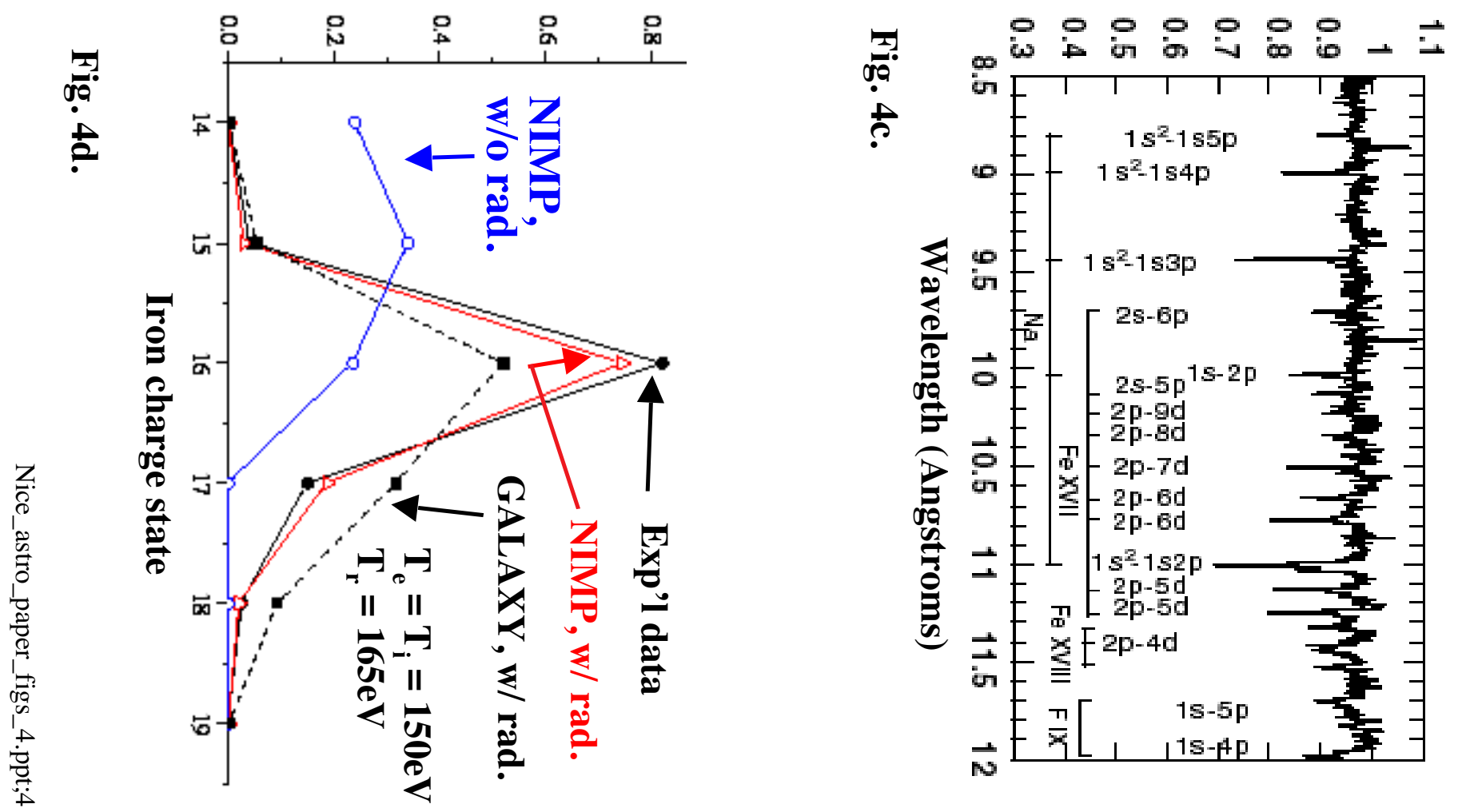